\title{
The PPE Pandemic: Sex-Related Discrepancies of N95 Mask Fit
}

\author{
Authors: \\ Laura Christopher, ${ }^{*}$ Theresa Rohr-Kirchgraber, ${ }^{2}$ Saralyn Mark ${ }^{3}$ \\ 1. Indiana University School of Medicine, Indianapolis, Indiana, USA \\ 2. Augusta University/University of Georgia Medical Partnership, Athens, \\ Georgia, USA \\ 3. iGIANT, Washington, District of Columbia, USA \\ *Correspondence to Theresa.RohrKirchgraber@UGA.EDU \\ Disclosure: $\quad$ The authors have declared no conflicts of interest. \\ Received: \\ 12.08 .20 \\ Accepted: \\ 06.07 .21 \\ Keywords: \\ COVID-19, gender, mask, personal protective equipment (PPE). \\ Citation: \\ EMJ Microbiol Infect Dis. 2021;2[1]:57-63. \\ Corrigendum: \\ This article was first published on $19^{\text {th }}$ August 2021. Since then a correction has been \\ made to the article. The corrigendum can be seen here.
}

\begin{abstract}
During the COVID-19 pandemic, caused by the severe acute respiratory syndrome coronavirus 2, healthcare professionals across the world have been at high risk of transmission because of their direct contact with infected patients. In October 2020, the International Council of Nurses (ICN) confirmed that 1,500 nurses had died from COVID-19 in 44 countries and estimated that healthcare worker COVID-19 fatalities worldwide could be more than 20,000. To ensure protection of healthcare personnel, properly fitting personal protective equipment (PPE) must be worn. In a 'one size fits all' world, the differences between the fit of PPE for men and women can have devastating consequences. An N95 respirator mask is a component of PPE outlined by the Centers for Disease Control and Prevention (CDC) for protection against COVID-19; however, N95 masks do not offer protection if they do not fit properly. Fit testing is performed to ensure an adequate seal of the mask on the wearer. A single-institution retrospective review was performed on fit testing results for male versus female wearers in an attempt to elucidate a difference in failure rates. Females failed at a significantly higher rate than their male counterparts $(6.67 \%$ female, $2.72 \%$ male; $p=\sim 0.22$ [with the continuity correction; 0.14 without it]), and the reason reported was often due to being 'small-boned' $(p<0.0001)$. Sex-related differences in proper PPE fit are not new; however, the COVID-19 pandemic has made the situation more acute, and sex-specific N95 mask designs must be developed quickly, as the pandemic shows little signs of abating.
\end{abstract}

\section{INTRODUCTION}

\section{Novel Coronavirus Disease 2019}

In December 2019, cases of pneumonia spiked in Wuhan, China. The cause of the acute respiratory illness was identified as a novel $\beta$-coronavirus.
This coronavirus was named the 2019-novel coronavirus on 12 January 2020 by the World Health Organization (WHO). On 11th February 2020 , the WHO officially named the respiratory disease as COVID-19 and the Coronavirus Study Group (CSG) of the International Committee proposed to name the new coronavirus, severe 
acute respiratory (SARS-CoV-2). ${ }^{1}$

syndrome

coronavirus-2

As cases increased, human-to-human transmission via respiratory droplets was recognised. ${ }^{2}$

Transmission to healthcare workers caring for infected patients was described on 20 January 2020, and the need for personal protective equipment (PPE) for healthcare professionals became imperative. The use of PPE such as the N95 masks became a standard need. Healthcare workers are at increased risk of infection from COVID-19 because of their close proximity with infected patients. Infection rates of healthcare workers have been reported as high as 29\% in China, 20\% in Italy, 6\% in the Netherlands, and $3 \%$ in the United States., ${ }^{3,4}$ In particular, healthcare workers from Black, Asian, and minority ethnic backgrounds are at increased risk of infection. ${ }^{5}$ As the pandemic continues, access to properly fitting PPE for healthcare workers is an important concern.

\section{Personal Protective Equipment}

The COVID-19 pandemic has caused a need for PPE like the world has never encountered before. The use of PPE reduces but does not fully eliminate the risk of transmission of highly infective agents. ${ }^{6}$ According to the Centers for Disease Control and Prevention (CDC), proper PPE for COVID-19 includes a face shield or goggles, a N95 or higher respirator, one pair of clean non-sterile gloves, and an isolation gown. ${ }^{7}$ The requirement for the use of N95 respirator masks is based on the current understanding of SARS-CoV-2 and related respiratory viruses. ${ }^{8}$ Current data suggests that likely routes of transmission of SARS-CoV-2 include close-range aerosol transmission by droplet and inhalation, and contact followed by self-inoculation via delivery to the eyes, nose, or mouth. ${ }^{8} \mathrm{~N} 95$ and higher-level respirators provide barrier and respiratory protection because of their tight fit and filtration characteristics; however, this fit must be assessed for effectiveness with a respirator fit test. ${ }^{8}$

Particulate respirators, such as N95 masks, have a non-woven fibrous filter media that captures particles. N95 masks have been shown to filter $99.8 \%$ of particles with a diameter of approximately $0.1 \mu \mathrm{m}$. SARS-
CoV-2 has a diameter of $0.1 \mu \mathrm{m}$, making N95 masks capable of filtering the viral particle..$^{9,10}$ In addition, N95 masks offer protection from the transmission of the virus by filtering respiratory droplets produced via coughing and sneezing.

Respirators are available in different models and sizes. Respirator fit testing requires selection of the most appropriate model. It includes the proper process of donning and requires at least 5 minutes of wear to assess comfort and effectiveness." The United States Department of Labor (DOL) reports the following criteria must be assessed to help determine the adequacy of respirator fit: chin properly placed, adequate strap tension, fit across nose bridge, respirator of proper size to space distance from nose to chin, tendency of respirator to slip, and selfobservation in mirror to evaluate fit and position." The test subject must then conduct a user seal test and undergo test exercises to determine if the fit is adequate. If the respirator doesn't fit properly, contaminated air can leak into the facepiece and potentially cause the wearer to breathe in hazardous substances. ${ }^{12}$

Proper respirator fit is vital for protection of the wearer. Previous reports have stated that facial hair, such as a beard, could prevent the mask from properly fitting; however, data regarding the differences in fit between male

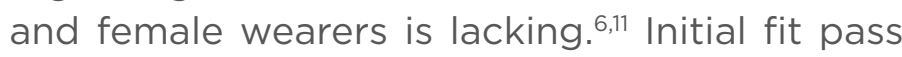
rates for filtering facepiece respirators vary widely, with lower pass rates found in women and Asians..$^{13}$ Higher initial fit pass rates were found in Caucasians (90\%) compared with Asians (84\%), and particularly low initial first pass rates were reported in Asian females, with a reported mean of $60 \% .^{14}$

Females, regardless of height and weight, have smaller bone structures than males, and most PPE has been designed for the male body, ${ }^{15}$ yet, according to the United States Census Bureau, in 2019, females held $76 \%$ of all healthcare jobs. $^{16,17}$ Improper fit of PPE can exacerbate the exposure to the virus by female healthcare and essential workers. A report published by the International Council of Nurses (ICN) on $3^{\text {rd }}$ June 2020 indicates that over 230,000 healthcare workers have contracted COVID-19, and more than 600 nurses have died from the virus. ${ }^{18}$ In another study investigating the characteristics 


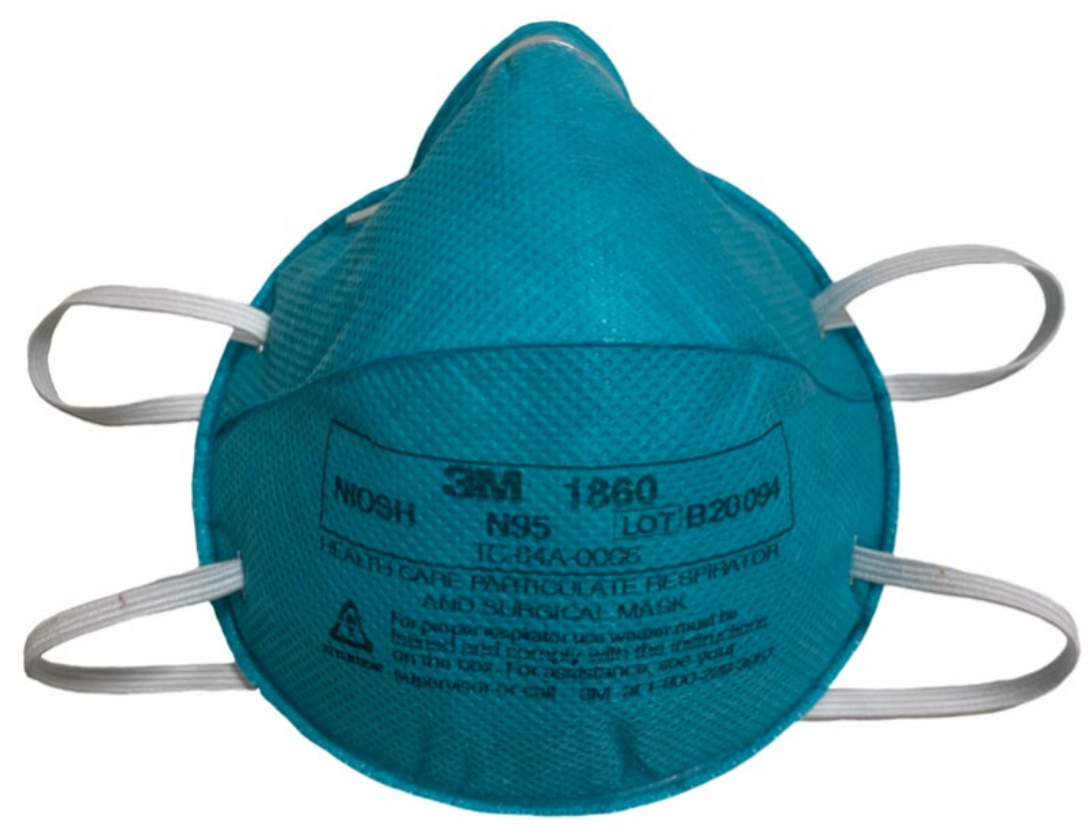

Figure 1: 3MMㅗ (Saint Paul, Minnesota, USA) Health Care Particulate Respirator and Surgical Mask 1860, N95 120 EA/ Case.

and related factors of COVID-19 infection in healthcare workers, it was found that 45 (29.80\%) infected healthcare workers were male and 106 (70.20\%) were female. ${ }^{19}$ In European Union (EU) countries, a higher proportion of healthcare workers diagnosed with COVID-19 infection were female. ${ }^{20}$

Additionally, in Spain, $72 \%$ of infected healthcare workers were female $(5,265)$ and in Italy $66 \%$ of infected healthcare workers were female $(10,657)$. Similar trends were found in the USA, with the CDC reporting $73 \%$ of infected healthcare workers were female. ${ }^{20}$ Therefore, it is important to ensure proper N95 fit for all healthcare workers, including females, since they make up the majority of the healthcare workforce.

\section{MATERIALS AND METHODS}

A retrospective review was performed on individuals who underwent respirator fit testing from December 2019 to June 2020 at a single institution. All fit testing included was performed by the staff at the health services centre on campus. The test uses an instrument to measure leakage around the face seal, resulting in a number called the 'fit factor'. The centre used Occupational Safety and Health (OSHA) test protocols." Quantitative data for fit testing was collected via the PortaCount Respirator FIT tester 8038 to determine the adequacy of the seal. Two different sized masks were available for fit testing (Model 3M1860 [3M; Saint Paul, Minnesota, USA), Regular/Small) (Figure 1). Fit test results were divided into two groups, male or female, and were evaluated for failure rates. Presented data is pooled from testing of both sizes. If the first mask size tested failed, the subsequent size was tested. If both sizes failed testing, then a failure and qualitative reason for failure was recorded. Reasons for fit test failures were reported as: large boned, small boned, facial asymmetry, facial hair, and/or no reason reported (Figure 2). Exclusion criterion included failure due to facial hair. A chi-squared analysis was performed on the data to determine statistical significance.

\section{RESULTS}

A total of 336 tests were performed during this time period. There were 34 failures for all males tested versus 13 failures for females. Of the 34 male failures, 31 were due to having facial hair, and were therefore excluded from the analysis.

The data analysed included 305 total respirator fit test results: 110 male and 195 female. Out of 


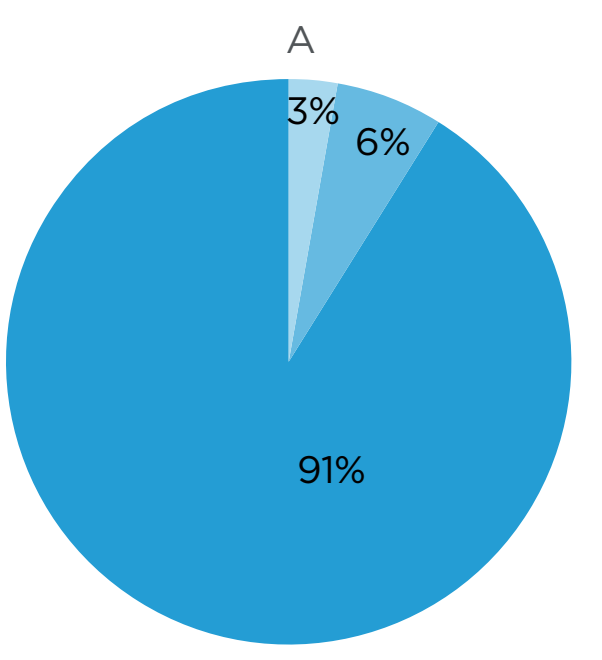

Facial hair

Large boned

Small boned

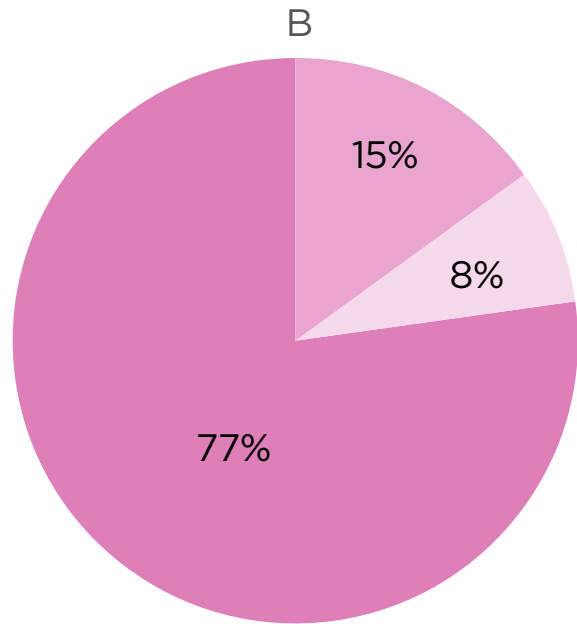

Facial asymmetry

Small boned

None reported

Figure 2: Sex-specific reasons for fit testing failures among A) male and B) female test subjects.

Reasons reported for respirator fit testing failures for both male and female test subjects, with their respective p-values. Failure reasons included facial hair, large boned, asymmetry, small boned, and no reason reported. Male failure total: 34 , with three due to non-facial hair reasons (large boned: $n=1 ;$ small boned: $n=2$ ). Female failure: 13 (facial asymmetry: $n=1$; small boned: $n=10$; none reported: $n=2$ ). Females failed significantly more due to being small boned when compared to male reasons for failure $(p<0.00001)$.

the 110 males, 107 passed fit testing and three failed, with a failure rate of $2.72 \%$. Out of the 195 females, 182 passed fit testing and 13 failed, with a failure rate of $6.67 \%$ (Table 1). Statistical analysis comparing expected versus observed outcomes of fit testing in males versus females indicated that females fail at higher rates than males $(p=\sim 0.22$ [with the continuity correction; 0.14 without it]) (Table 1).

\section{DISCUSSION}

Improper sizing of PPE is not a new problem; however, the COVID-19 pandemic has made the situation more acute. This study was designed to determine statistical significance of the fit of N95 respirator masks, an important piece of PPE for COVID-19 protection, comparing male and female wearers.

Proper fit of PPE for healthcare workers is crucial for protection during the COVID-19

Table 1: Total of 305 fit tests performed and analysed: 110 male and 195 female. Failure rates for male and female test subjects were $2.72 \%$ and $6.67 \%$, respectively ( $p=\sim 0.22$ [with the continuity correction; 0.14 without it]).

\begin{tabular}{|r|c|c|c|}
\hline & Male (facial hair included) & Male (facial hair excluded) & Female \\
\hline Pass & 141 & 107 & 182 \\
\hline Fail & 34 & 3 & 13 \\
\hline Failure Rates & $31.80 \%$ & $2.72 \%$ & $6.67 \%$ \\
\hline p-value & NA & $\mathrm{p}=\sim 0.22$ (with the continuity correction; 0.14 without it) \\
\hline
\end{tabular}

NA: not applicable. 
pandemic, not just for the wearer but also for patients. Ineffectiveness of PPE may contribute to nosocomial transmission of COVID-19.6,21 Compared with the general population, healthcare workers have an increased risk of testing positive for COVID-19, even after adjusting for testing eligibility. ${ }^{22}$ Compared with non-healthcare workers, healthcare workers were younger (43 versus 53 years; $p<0.001$ ) and more likely female (118/193 [61\%] versus 1,211/2,649 [46\%]; $p<0.001) .{ }^{23}$

One cluster randomised study attempted to compare non-fitted N95 masks versus fitted N95 masks and found that all outcomes (clinical respiratory illness, influenza-like illness, laboratory confirmed respiratory viral infection, and influenza) were consistently lower in the fitted N95 group. ${ }^{24}$ In the present study, male wearers failed fit testing at a significantly lower rate than females $(2.72 \%$ male, $6.67 \%$ female; $p=\sim 0.22$ [with the continuity correction; 0.14 without it]) (Table 1). Currently, N95 masks and other PPE equipment are made in 'one size fits all' and do not consider the differences that facial structure due to sex can have on the fit.

Reasons for male fit testing failure due to facial hair were excluded because numerous studies have shown that beard growth at points where the face and respirator come in contact prevents a good seal. ${ }^{25,26}$ In fact, a study assessing the impact of facial hair on quantitative respirator fit in male healthcare workers showed that no full-bearded healthcare workers achieved an adequate fit, and adequate fit decreased significantly with increasing facial hair $(p<0.01$ for trend). ${ }^{26}$ Therefore, if the wearer chooses to maintain facial hair, the OSHA recommends the use of respirators that do not rely on a tight facepiece seal between the respirator inlet covering and the skin (i.e., loose-fitting helmets or hoods). ${ }^{25}$ However, as previously mentioned, facial hair is a choice, while face shape is genetically determined.

Sex-related differences in PPE fit are widespread across many different fields, not just healthcare. In 2016, poor access to appropriately fitting PPE was highlighted in a study of female construction workers. Female labourers, carpenters, and ironworkers were enrolled in semi-structured focus groups in New York City, USA. The majority reported fit problems for many types of PPE (e.g., gloves, harnesses, safety vest, work boots, and outerwear), noting that the equipment provided was too large. ${ }^{27}$ The authors concluded that female construction workers have difficulty accessing properly fitting PPE that is designed for women. ${ }^{27}$ A UK survey performed in May 2016 by the trade union Prospect, Women in Science and Engineering, the Trades Union Congress, and the Institution of Mechanical Engineers found that ill-fitting PPE was common but often accepted as 'part of the job'.15

Many responses from PPE fit surveys indicate that improperly fitting PPE hinder female's ability to do their work. ${ }^{15,27,28}$ The challenge at hand is that PPE has been developed to fit a male frame, with smaller sizing being provided for females. However, females are not just smaller versions of men. A comparison of male and female body sizes and proportions performed in 1977 on military personnel indicated that even females of equal height and weight to their male counterparts do not have the same body proportions. ${ }^{29}$ Unsurprisingly, facial dimensions vary with both sex and ethnic groups. A study focusing on the impact of race and ethnicity upon the development of PPE in the USA workforce showed that sex and race/ethnicity significantly differ. ${ }^{30}$ African Americans have statistically shorter, wider, and shallower noses than Caucasians. ${ }^{30}$ While Hispanic workers have 14 facial features that are larger than Caucasians, their nose protrusion, height, and head length are significantly shorter. ${ }^{30}$ The final ethnic group analysed was composed of primarily Asian subjects that had statistically different dimensions from Caucasians for 16 of the anthropometric values analysed..$^{30}$ In addition, sex also significantly contributed to the size of facial features. ${ }^{30,31}$ Even though two N95 mask sizes were offered at this institution, regular and small, females still failed for being small-boned at a significant rate $(p<0.00001)$ due to these sex-related differences in facial dimensions (Figure 2).

A recent study also found that racial and ethnic differences throughout the pandemic 
have contributed to the patterns of infection among healthcare workers. ${ }^{5}$ Noting that healthcare workers that were Black, Asian, or Hispanic were over 3-fold more likely to contract COVID-19 compared to Caucasian healthcare workers; it is postulated that this increased incidence among underrepresented minority groups may be due to improper PPE fit. However, data is limited on this subject and this is perhaps another field for further study and another consideration in the push to redesign PPE. Facial differences by sex and gender, as well as racial and ethnic variation, lead to poorly fitting masks and may be a contributing factor to this disparity.

The Personal Protective Equipment Regulations 2002 and the Personal Protective Equipment at Work Regulations 1992 (as amended) place a legal requirement on employers to provide PPE to workers if it is needed to protect them from any workplace hazards to their safety or health. ${ }^{28}$ Employers must ensure that the PPE is suitable for the purpose, causing significant concerns for appropriate fitting N95 masks for women health care workers. ${ }^{28}$ The unisex approach to PPE negatively affects both the work and safety of female wearers. PPE is intended to provide protection, yet currently may be ineffective for more than $75 \%$ of the healthcare workforce. The results of this study indicate that there is a disparity in the fit of N95 masks for female wearers. Further sex-specific studies should be performed with larger sample sizes, including various ethnicities, and other proponents of PPE to ensure both statistical and clinical significance.

\section{CONCLUSION}

The COVID-19 pandemic has caused a PPE pandemic. With over 49.7 million cases worldwide and over 1.27 million deaths as of November 2020 , the pandemic shows little signs of natural abatement. ${ }^{32}$ Now, more than ever, it is important for healthcare workers to have properly fitting PPE to ensure their health and safety and the safety of those around them. In many countries, N95 respirator masks, as outlined by the CDC, are part of PPE for protection against COVID-19, but these same essential workers are faced with wearing masks that do not properly fit, leaving them at increased risk for infection. N95 masks were not designed for female facial dimensions and, because of inadequate fit, female healthcare workers are not properly protected. Developing a sex-specific approach to designing N95 masks is vital as it would protect both healthcare workers and the patients they serve.

\section{References}

1. Guo YR et al. The origin, transmission and clinical therapies on coronavirus disease 2019 (COVID-19) outbreak an update on the status. Mil Med Res. 2020;7(1):1-10.

2. Singhal T. A review of coronavirus disease-2019 (COVID-19). Indian J Pediatr. 2020;87(4):281-6.

3. Sommerstein $R$ et al. Risk of SARSCoV-2 transmission by aerosols, the rational use of masks, and protection of healthcare workers from COVID-19. Antimicrob Resist Infect Control. 2020;9(1):100.

4. The Lancet. COVID-19: protecting health-care workers. Lancet. 2020;395(10228):922

5. Nguyen NS et al.; Coronavirus Pandemic Epidemiology Consortium. Risk of COVID-19 among front-line health-care workers and the general community: a prospective cohort study. Lancet Public Health. 2020;5:e475-83.

6. Ağalar C, Engın DÖ. Protective measures for COVID-19 for healthcare providers and laboratory personnel. Turk J Med Sci. 2020;50(SI-1):578-84.

7. Centers for Disease Control and Prevention (CDC). Interim infection prevention and control recommendations for healthcare personnel during the coronavirus disease 2019 (COVID-19) pandemic. 2020 Available at: https://www.cdc.gov/ coronavirus/2019-ncov/hcp/infection-control-recommendations.html. Last accessed: 20 July 2021.

8. Centers for Disease Control and Prevention (CDC). Using personal protective equipment (PPE). 2020. Available at: https://www.cdc.gov/coronavirus/2019-ncov/hcp/using-ppe.html. Last accessed: 20 July 2021.

9. Johnson E. Filtration mechanisms of particulate respirators. 2005. Available at: https://multimedia.3m.com/ mws/media/4103640/filtration-mechanisms-for-particulate-respirators.pdf. Last accessed: 5 October 2020.

10. Bar-On YM et al. Science forum:
SARS-CoV-2 (COVID-19) by the numbers. eLife. 2020;9:e57309.

11. Occupational Safety and Health Administration (OSHA). Fit testing procedures (mandatory). Appendix A to \$1910.134-fit testing procedures (mandatory). 2004. Available at: https://www.osha.gov/ laws-regs/regulations/standardnumber/1910/1910.134AppA. Last accessed: 5 October 2020.

12. Occupational Safety and Health Administration (OHSA). Transcript for the OSHA training video entitled respirator fit testing. 2012. Available at: https://www.osha.gov/video/respiratory-protection/fit-testing/transcript. Last accessed: 20 July 2021.

13. Wilkinson IJ et al. Evaluation of a large-scale quantitative respirator-fit testing program for healthcare workers: survey results. Infect Control Hosp Epidemiol. 2010;31(9):918-25.

14. Han DH, Choi KL. Facial dimensions and predictors of fit for half-mask respirators in Koreans. AIHA J (Fair- 
fax, Va). 2003;64(6):815-22.

15. Ghani R. Is PPE working for women? The problem of ill-fitting personal protective equipment of women in industry. Occupational Health at Work. 2017;13(6):32-5.

16. Pugh R. COVID-19 PPE gender divide: no one-size-fits-all? 2020. Available at: https://www.medscape.com/viewarticle/929860?nlid=135399 5653\&src=wnl_newsalrt_daily_200504_ MSCPEDIT\&uac $=364031 \mathrm{CJ} \& \mathrm{im}-$ $\mathrm{pID}=2369864 \& \mathrm{faf}=1 \# \mathrm{vp}$ _1. Last accessed: 5 October 2020.

17. Cheeseman Day J, Christnacht C. Your health care is in women's hands. 2019. Available at: https://www.census.gov/library/stories/2019/08/yourhealth-care-in-womens-hands.html. Last accessed: 5 October 2020.

18. The International Council of Nurses (ICN). More than 600 nurses die from COVID-19 worldwide. 2020. Available at: https://www.icn.ch/news/more600-nurses-die-covid-19-worldwide. Last accessed: 5 October 2020.

19. Lai $X$ et al. What influences the infection of COVID-19 in healthcare workers? J Infect Dev Ctries. 2020;14(11):1231-7.

20. Miyamoto I. Covid-19 healthcare workers: $70 \%$ are women. 2020. Available at: https://www.jstor.org/ stable/resrep24863?seq=1\#metada- ta_info tab contents. Last accessed: 5 October 2020

21. Tran $\mathrm{K}$ et al. Aerosol generating procedures and risk of transmission of acute respiratory infections to healthcare workers: a systematic review. PLoS One. 2012;7(4):e35797.

22. Regli $A$ et al. The role of fit testing N95/FFP2/FFP3 masks: a narrative review. Anaesthesia. 2021;76(1):91100.

23. Kim R et al. Comparison of COVID-19 infections among healthcare workers and non-healthcare workers. PLoS One. 2020;15(12):e0241956.

24. Maclntyre $C R$ et al. A cluster randomized clinical trial comparing fit-tested and non-fit-tested N95 respirators to medical masks to prevent respiratory virus infection in healthcare workers. Influenza Other Respir Viruses. 2011;5:170-9.

25. Occupational Safety and Health Administration (OSHA). Standard interpretations: inquiry on beards, respirator use, and fit testing of respirators. 1996. Available at: https:// www.osha.gov/laws-regs/standardinterpretations/1996-10-03. Last accessed: 23 July 2021.

26. Sandaradura I et al. A close shave? Performance of $\mathrm{P} 2 / \mathrm{N} 95$ respirators in health care workers with facial hair: results of the BEARDS (Adequate Respiratory Defences) study. J Hosp Infect. 2020;104(4):529-33.

27. Onyebeke LC et al. Access to properly fitting personal protective equipment for female construction workers. Am J Ind Med. 2016;59(11):1032-40.

28. Trades Union Congress (TUC) Personal protective equipment and women. 2017. Available at: https:// www.tuc.org.uk/sites/default/files/ PPEandwomenguidance.pdf. Last accessed: 5 October 2020.

29. Robinette $\mathrm{K}$ et al. A comparison of male and female body sizes and proportions (1979), Yellow Springs, $\mathrm{OH}$ : Anthropology Research Project Inc.

30. Zhuang $Z$ et al. Facial anthropometric differences among gender, ethnicity, and age groups. Ann Occup Hyg. 2010;54(4):391-402.

31. Zhuang Z, Bradtmiller B. Head-andface anthropometric survey of U.S. respirator users. J Occup Environ Hyg. 2005;2(11):567-76.

32. World Health Organization. Weekly epidemiological update - 10 November 2020. 2020. Available at: https://www.who.int/publications/m/item/weekly-epidemiological-update---10-november-2020. Last accessed: 20 July 2021. 